\title{
Spinning Skyrmions and the Skyrme Parameters
}

\author{
Richard A. Battye ${ }^{1}$, Steffen Krusch ${ }^{2}$ and Paul M. Sutcliffe ${ }^{2}$ \\ ${ }^{1}$ Jodrell Bank Observatory, Macclesfield, Cheshire SK11 9DL U.K. \\ \& School of Physics and Astronomy, Schuster Laboratory, \\ University of Manchester, Brunswick St, Manchester M13 9PL, U.K. \\ Email : rbattye@jb.man.ac.uk \\ ${ }^{2}$ Institute of Mathematics, University of Kent, Canterbury, CT2 7NF, U.K. \\ Email : S.Krusch@kent.ac.uk \\ Email : P.M.Sutcliffe@kent.ac.uk
}

July 2005

\begin{abstract}
The traditional approach to fixing the parameters of the Skyrme model requires the energy of a spinning Skyrmion to reproduce the nucleon and delta masses. The standard Skyrme parameters, which are used almost exclusively, fix the pion mass to its experimental value and fit the two remaining Skyrme parameters by approximating the spinning Skyrmion as a rigid body. In this paper we remove the rigid body approximation and perform numerical calculations which allow the spinning Skyrmion to deform and break spherical symmetry. The results show that if the pion mass is set to its experimental value then the nucleon and delta masses can not be reproduced for any values of the Skyrme parameters; the commonly used Skyrme parameters are simply an artifact of the rigid body approximation. However, if the pion mass is taken to be substantially larger than its experimental value then the nucleon and delta masses can be reproduced. This result has a significant effect on the structure of multi-Skyrmions.
\end{abstract}




\section{Introduction}

The Skyrme model [13] is a nonlinear theory of pions which is an approximate, low energy effective theory of quantum chromodynamics, obtained in the limit of a large number of quark colours [15]. Skyrmions are topological soliton solutions of the model and are candidates for an effective description of nuclei, with an identification between soliton and baryon numbers.

The Lagrangian of the Skyrme model contains only three free parameters; two of these set the energy and length units and the third corresponds to the (tree-level) pion mass. In Ref.[2] the energy and length units were calculated by fitting to the masses of the nucleon and delta resonance assuming massless pions, and in Ref.[1] this calculation was repeated using the experimental value for the pion mass. This set of parameters is invariably used in the study of the Skyrme model, and so we shall refer to them as the standard values.

Recently [4] the properties of classical multi-Skyrmions have been investigated for a range of Skyrme parameters and it has been found that important qualitative differences arise as the Skyrme parameters are varied. In particular, setting the pion mass to be substantially larger than its experimental value yields multi-Skyrmions whose qualitative features appear to be closer to those of real nuclei. For example, the size of nuclei scales with mass number $A$ like $A^{1 / 3}$ rather than like $\sqrt{A}$, and the stability properties of small nuclei with $A=5$ and $A=8$ also seem more realistic. These results provide motivation to re-examine the original work in Refs. $[2,1]$ where the standard Skyrme parameters were first calculated.

The approach of Refs.[2, 1] involves the zero-mode quantization of a single Skyrmion as a rigid body. This is effectively a study of spinning Skyrmions which assumes that a Skyrmion does not deform as it spins. Early papers [5, 11] pointed out the limitations of this approximation and improved upon it by allowing the Skyrmion to deform within a spherically symmetric hedgehog ansatz. Including only this deformation already reveals that with the experimental value of the pion mass there are now no values of the Skyrme parameters that can fit the masses of both the nucleon and delta. Thus, the standard Skyrme parameters are simply an artifact of the rigid body approximation.

The need to take into account the deformation of a spinning Skyrmion, and in particular to allow deformations which break spherical symmetry, has been noted by several authors $[12,7]$ using a range of different physical perspectives. In this paper we address this issue by performing numerical computations of spinning Skyrmions in the full field theory assuming only an axial symmetry. We compute the energies of spinning Skyrmions for a range of Skyrme parameters and pion masses, and find that the nucleon and delta masses can be fit to the experimental values only if the pion mass is taken to be larger than twice the experimental value. A larger value for the tree-level pion mass is not necessarily in conflict with the smaller experimental value, as we discuss in the next section. 


\section{Spinning Skyrmions}

The field of the Skyrme model [13] is an $S U(2)$-valued scalar $U$. It is convenient to introduce the $s u(2)$-valued current $R_{\mu}=\left(\partial_{\mu} U\right) U^{\dagger}$ and write the Lagrangian as

$$
L=\int\left\{-\frac{F_{\pi}^{2}}{16} \operatorname{Tr}\left(R_{\mu} R^{\mu}\right)+\frac{1}{32 e^{2}} \operatorname{Tr}\left(\left[R_{\mu}, R_{\nu}\right]\left[R^{\mu}, R^{\nu}\right]\right)+\frac{m_{\pi}^{2} F_{\pi}^{2}}{8} \operatorname{Tr}(U-1)\right\} d^{3} x .
$$

Here $F_{\pi}, e$ and $m_{\pi}$ are parameters, whose values are fixed by comparison with experimental data. $F_{\pi}$ may be interpreted as the pion decay constant, $e$ is a dimensionless constant and $m_{\pi}$ has the interpretation of the tree-level pion mass (we use units in which $\hbar$ is one). The experimental values for the pion decay constant and pion mass are $F_{\pi}=186 \mathrm{MeV}$ and $m_{\pi}=138 \mathrm{MeV}$ respectively.

The approach of Refs. $[2,1]$ is to fix $F_{\pi}$ and $e$ by fitting the energies of a quantized Skyrmion to the masses of the nucleon and delta resonance, assuming either massless pions [2] or the experimental value for the pion mass [1], yielding the standard values $m_{\pi}=138 \mathrm{MeV}, F_{\pi}=108 \mathrm{MeV}$ and $e=4.84$. Note that this value of $F_{\pi}$ is substantially lower than the experimental value, but if $F_{\pi}$ and $m_{\pi}$ are both fixed to the experimental values then the only free parameter is $e$, and this does not allow a simultaneous fit for both the nucleon and delta masses.

The quantization of the Skyrme model is difficult due to the fact that it is a nonrenormalizable field theory. One optimistic approach is that these effects can be partially modeled by a renormalization of the Skyrme parameters, in which case $F_{\pi}$ and $m_{\pi}$ could be interpreted as the renormalized pion decay constant and the renormalized pion mass. Therefore values which differ from the experimental values are not necessarily a contradiction. Furthermore, we are mainly interested in reproducing the properties of nuclei for all nucleon numbers and are willing to place less emphasis on matching the pion physics if necessary. In this paper we shall treat the three Skyrme parameters $F_{\pi}, e$ and $m_{\pi}$ all as free parameters, and indeed we find that neither $F_{\pi}$ nor $m_{\pi}$ can be set to their experimental value if the nucleon and delta masses are to be fit, once the deformation of a spinning Skyrmion is taken into account.

It is useful to rescale the spacetime coordinates by $x_{\mu} \mapsto 2 x_{\mu} /\left(e F_{\pi}\right)$ so that the relative coefficient between the first two terms in (2.1) is independent of the Skyrme parameters. After applying this rescaling the Lagrangian becomes

$$
L=\frac{F_{\pi}}{4 e} \int\left\{-\frac{1}{2} \operatorname{Tr}\left(R_{\mu} R^{\mu}\right)+\frac{1}{16} \operatorname{Tr}\left(\left[R_{\mu}, R_{\nu}\right]\left[R^{\mu}, R^{\nu}\right]\right)+m^{2} \operatorname{Tr}(U-1)\right\} d^{3} x
$$

where we have introduced the rescaled pion mass $m=2 m_{\pi} /\left(F_{\pi} e\right)$. From this form of the Lagrangian it is now clear that the parameter combinations $F_{\pi} /(4 e)$ and $2 /\left(e F_{\pi}\right)$ merely determine the energy and length units of classical static Skyrmions. However, the rescaled pion mass $m$ can not be scaled away and so can have an effect on Skyrmion structure. A recent study [4] has demonstrated that significant qualitative differences arise for multiSkyrmions as the parameter $m$ is varied, so the determination of the parameters of the 
Skyrme model (and in particular $m$ ) is important. Note that, in the quantization of a Skyrmion we must consider its spin, and then the parameters $F_{\pi}$ and $e$ can not simply be scaled away.

For a classical static Skyrmion the energy derived from the Lagrangian (2.2) is

$$
E_{0}=\frac{F_{\pi}}{4 e} \int\left\{-\frac{1}{2} \operatorname{Tr}\left(R_{i} R_{i}\right)-\frac{1}{16} \operatorname{Tr}\left(\left[R_{i}, R_{j}\right]\left[R_{i}, R_{j}\right]\right)+m^{2} \operatorname{Tr}(1-U)\right\} d^{3} x .
$$

The standard approach of Refs.[2,1] is to quantize the rotational zero modes of the Skyrmion as a rigid body, which yields multiplets with equal spin and isospin in each multiplet. The details are as follows.

In spherical polar coordinates $(r, \theta, \phi)$ the ansatz for a hedgehog Skyrmion rigidly rotating around the $\phi$-axis is

$$
U=\cos f+i \sin f\left(\tau_{3} \cos \theta+\sin \theta\left(\tau_{1} \cos (\phi+\omega t)+\tau_{2} \sin (\phi+\omega t)\right)\right)
$$

where $\tau_{i}$ denote the Pauli matrices, $f(r)$ is the radial profile function (with boundary conditions $f(0)=\pi$ and $f(\infty)=0$ ) and $\omega$ is the rotation frequency in the rescaled coordinates; note that the rotation frequency in physical units is given by $\Omega=\omega e F_{\pi} / 2$.

Substituting this ansatz into the Lagrangian (2.2) gives

$$
L=\frac{1}{2} \Lambda \Omega^{2}-E_{0}
$$

where $E_{0}$ is the static energy

$$
E_{0}=\frac{\pi F_{\pi}}{e} \int_{0}^{\infty}\left\{r^{2} f^{\prime 2}+2 \sin ^{2} f\left(1+f^{\prime 2}\right)+\frac{\sin ^{4} f}{r^{2}}+2 m^{2} r^{2}(1-\cos f)\right\} d r
$$

and $\Lambda$ is the moment of inertia

$$
\Lambda=\frac{16 \pi}{3 e^{3} F_{\pi}} \int_{0}^{\infty}\left\{\sin ^{2} f\left(r^{2}\left(1+f^{\prime 2}\right)+\sin ^{2} f\right)\right\} d r .
$$

The equation for the profile function which follows from the Lagrangian (2.5) can equivalently be obtained my minimizing the total energy

$$
E=E_{0}+\frac{J^{2}}{2 \Lambda}
$$

where $J=\Omega \Lambda$ is the spin, which is a conserved quantity.

The classical Skyrmion is quantized within the Bohr framework by requiring the spin to be quantized as $J^{2}=j(j+1)$, where $j$ is the spin quantum number taking values $j=\frac{1}{2}$ for the nucleon and $j=\frac{3}{2}$ for the delta. Thus, within the classical picture, the nucleon and delta are simply spinning Skyrmions with a particular rotation frequency.

In the above discussion we have assumed that the spinning Skyrmion remains spherically symmetric. In the treatment of Refs. $[2,1]$ a further approximation is employed, 
namely that the Skyrmion does not deform at all when it spins. In this approach the profile function is not determined by minimization of the total energy (2.8), but rather by minimization of only the static energy $E_{0}$. The additional contribution to the energy due to the spin is then calculated given the static energy minimizing profile function. A crucial point is that this rigid body approximation allows any value of the spin to be obtained for a spinning Skyrmion, since one simply sets the rotation frequency to be $\Omega=J / \Lambda$, where $\Lambda$ is the moment of inertia of the static Skyrmion.

Setting the pion mass to its experimental value $\left(m_{\pi}=138 \mathrm{MeV}\right)$ leaves the remaining parameters $F_{\pi}$ and $e$ to be determined by fixing the energy of the $j=\frac{1}{2}$ and $j=\frac{3}{2}$ spinning Skyrmions to the masses of the nucleon $\left(M_{N}=939 \mathrm{MeV}\right)$ and $\operatorname{delta}\left(M_{\Delta}=1232 \mathrm{MeV}\right)$. In Fig. 1 we plot, for a range of $e$, the value of $F_{\pi}$ required to fit the nucleon mass (solid curve) and the value of $F_{\pi}$ required to fit the delta mass (dashed curve) using the rigid body approximation. These two curves cross at the value $e=4.84$ with $F_{\pi}=108 \mathrm{MeV}$, which are the standard parameter values obtained in Ref.[1] and produce a rescaled pion mass $m=0.526$.

The limitations of the rigid body approximation were highlighted in the papers $[5,11]$ and a first improvement was made by allowing the Skyrmion to deform within a spherically symmetric hedgehog ansatz. In this approach the profile function $f(r)$ is determined by minimization of the total energy (2.8). An analysis of the ordinary differential equation for the profile function reveals that a localized finite energy solution exists only if the rotation frequency satisfies the constraint $\Omega^{2} \leq \frac{3}{2} m_{\pi}^{2}$. Furthermore, at the maximal rotation frequency $\Omega=\sqrt{\frac{3}{2}} m_{\pi}$ the moment of inertia $\Lambda$ is finite so there is an upper bound on the spin $J$. As the Skyrme parameter $e$ is increased there is a point at which the required spin for the delta (or nucleon) exceeds the upper bound, so there is no spinning Skyrmion that describes the delta (or nucleon). In Refs. [5, 11] it was found that with the experimental value of the pion mass the standard values for $e$ and $F_{\pi}$ are in the region for which there is no spinning Skyrmion solution for the delta, so the standard Skyrme parameters are simply an artifact of the rigid body approximation and there are no Skyrme parameters that can be chosen to fit the masses of both the nucleon and delta.

Physically one expects the constraint on the spin to be $\Omega^{2} \leq m_{\pi}^{2}$, since this expresses the fact that the Skyrmion can only spin at a frequency upto the pion mass before it begins to radiate pions. The extra factor of $\sqrt{\frac{3}{2}}$ in the constraint for the spinning hedgehog field reflects the fact that the radiation is assumed to be a spherical wave in the hedgehog approximation. A consistent ansatz for a spinning Skyrmion can have at most an axial symmetry and will radiate differently in the directions orthogonal and parallel to the symmetry axis. Thus, although the deformed hedgehog approximation captures the qualitative feature that there is a maximal spin, the quantitative details require the computation of spinning Skyrmions with axial symmetry.

A limited numerical study (using small grids) of axially symmetric spinning Skyrmions has been performed [14], but for only one set of Skyrme parameters, so the dependence on the Skyrme parameters that we wish to address in this study has not been investigated.

Introducing cylindrical polar coordinates $\rho, \chi, z$ the ansatz for an axially symmetric 
spinning Skyrmion is a simple generalization of the one introduced in Ref.[8] and is given by

$$
U=\psi_{3}+i \psi_{2} \tau_{3}+i \psi_{1}\left(\tau_{1} \cos (\chi+\omega t)+\tau_{2} \sin (\chi+\omega t)\right)
$$

where $\boldsymbol{\psi}(\rho, z)=\left(\psi_{1}, \psi_{2}, \psi_{3}\right)$ is a three-component unit vector that is independent of $\chi$. The boundary conditions on $\boldsymbol{\psi}$ are that $\boldsymbol{\psi} \rightarrow(0,0,1)$ as $\rho^{2}+z^{2} \rightarrow \infty$, and on the symmetry axis $\rho=0$ we require $\psi_{1}=0$ and $\partial_{\rho} \psi_{2}=\partial_{\rho} \psi_{3}=0$.

With the axial ansatz (2.9) the expressions for the static energy and moment of inertia become

$$
\begin{gathered}
E_{0}=\frac{\pi F_{\pi}}{2 e} \int\left\{\left(\partial_{\rho} \boldsymbol{\psi} \cdot \partial_{\rho} \boldsymbol{\psi}+\partial_{z} \boldsymbol{\psi} \cdot \partial_{z} \boldsymbol{\psi}\right)\left(1+\frac{\psi_{1}^{2}}{\rho^{2}}\right)+\left|\partial_{\rho} \boldsymbol{\psi} \times \partial_{z} \boldsymbol{\psi}\right|^{2}+\frac{\psi_{1}^{2}}{\rho^{2}}+2 m^{2}\left(1-\psi_{3}\right)\right\} \rho d \rho d z \\
\Lambda=\frac{4 \pi}{e^{3} F_{\pi}} \int\left\{\psi_{1}^{2}\left(\partial_{\rho} \boldsymbol{\psi} \cdot \partial_{\rho} \boldsymbol{\psi}+\partial_{z} \boldsymbol{\psi} \cdot \partial_{z} \boldsymbol{\psi}+1\right)\right\} \rho d \rho d z
\end{gathered}
$$

For a given spin $J=\sqrt{j(j+1)}$ the configuration which minimizes the total energy (2.8) needs to be computed, with $E_{0}$ and $\Lambda$ given by (2.10) and (2.11). This minimization is performed numerically using a simulated annealing algorithm on a grid in the $(\rho, z)$-plane containing $250 \times 500$ grid points and a lattice spacing of 0.06 .

With the pion mass set to its experimental value of $m_{\pi}=138 \mathrm{MeV}$, we calculate, for a range of the Skyrme parameter $e$, the value of $F_{\pi}$ required to match the energy of a $j=\frac{1}{2}$ spinning Skyrmion to the nucleon mass and also the value of $F_{\pi}$ required to match the $j=\frac{3}{2}$ spinning Skyrmion energy to the delta mass.

The results of the simulated annealing minimization are displayed in Fig. 1, where circles denote the values required for the nucleon fit and squares denote the delta fit. Note that the results of the axial calculation (circles and squares) are extremely close to those of the rigid body approximation (solid and dashed curves), indicating that for this value of the pion mass there is little deformation of the spinning Skyrmion. However, the crucial point is that the spinning Skyrmion exists only if the parameter $e$ is less than a critical value, which is why the axial data in Fig. 1 terminates. At the termination point, the rotation frequency $\Omega$, which we calculate as $J / \Lambda$, is equal to the pion mass $m_{\pi}$ and therefore the Skyrmion has attained its maximal value of the spin. If $e$ is increased beyond this value the Skyrmion can not be spun fast enough to reach the required spin $J$ with the new parameters, and so the required spinning Skyrmion solution does not exist. Clearly, since the spin of the delta is greater than that of the nucleon the termination point for the delta is at a lower value of $e$ than for the nucleon.

A linearization of the $\psi_{1}$ field equation yields

$$
\partial_{i} \partial_{i} \psi_{1}-\left(m^{2}-\omega^{2}\right) \psi_{1}=0
$$

so a finite energy solution exists only if the effective mass is non-negative ie. $\omega^{2} \leq m^{2}$, or in physical units $\Omega^{2} \leq m_{\pi}^{2}$, which is the criterion we have used to identify the termination point of the spinning Skyrmion. On a finite numerical grid all configurations have finite energy so care has to be taken in determining the termination point. It would be easy to 


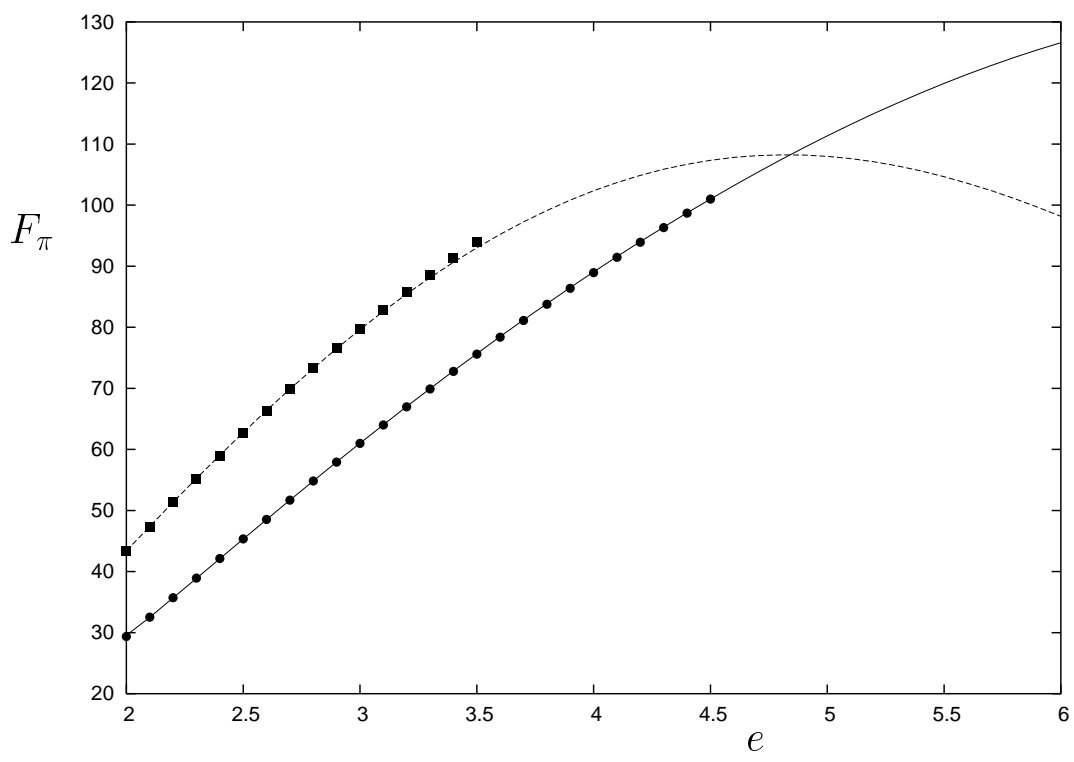

Figure 1: For $m_{\pi}=138 \mathrm{MeV}$, the graph displays, as a function of the Skyrme parameter $e$, the value of $F_{\pi}$ required to fit the nucleon mass (solid curve for rigid body approximation and circles for axial deformation) and delta mass (dashed curve for rigid body approximation and squares for axial deformation). Note that the data for the axial deformation of the nucleon and delta do not cross.

mistakenly compute a configuration with a rotation frequency which is apparently greater than $m_{\pi}$, but one can check that for such a configuration the computed energy is not independent of the size of the numerical grid, which signals that this is not a finite energy solution.

The crucial point about the axial data in Fig. 1 is that there is no crossing point of the nucleon and delta data, so there are no values of $e$ and $F_{\pi}$ that fit both the nucleon and delta masses. The crossing point within the rigid body approximation is well beyond the termination points of the axial data, so the standard values are an artifact of the approximation and do not correspond to any spinning Skyrmion solutions.

If the nucleon and delta masses are to be matched to the energies of spinning Skyrmions then the only possibility is to increase the pion mass beyond its experimental value. Increasing $m_{\pi}$ allows the Skyrmion to spin at a greater frequency, so larger values of $e$ can be attained, and it may be possible that both masses can be fit simultaneously.

It is of interest to note that several other studies, from different perspectives, have indicated that a pion mass larger than the experimental value yields Skyrmion results that agree better with experimental data. For example, studies of the Roper resonance [6] and comparisons between vibrational frequencies of multi-Skyrmions and nuclear gamma ray spectra [3], produce improved results with a larger pion mass. 


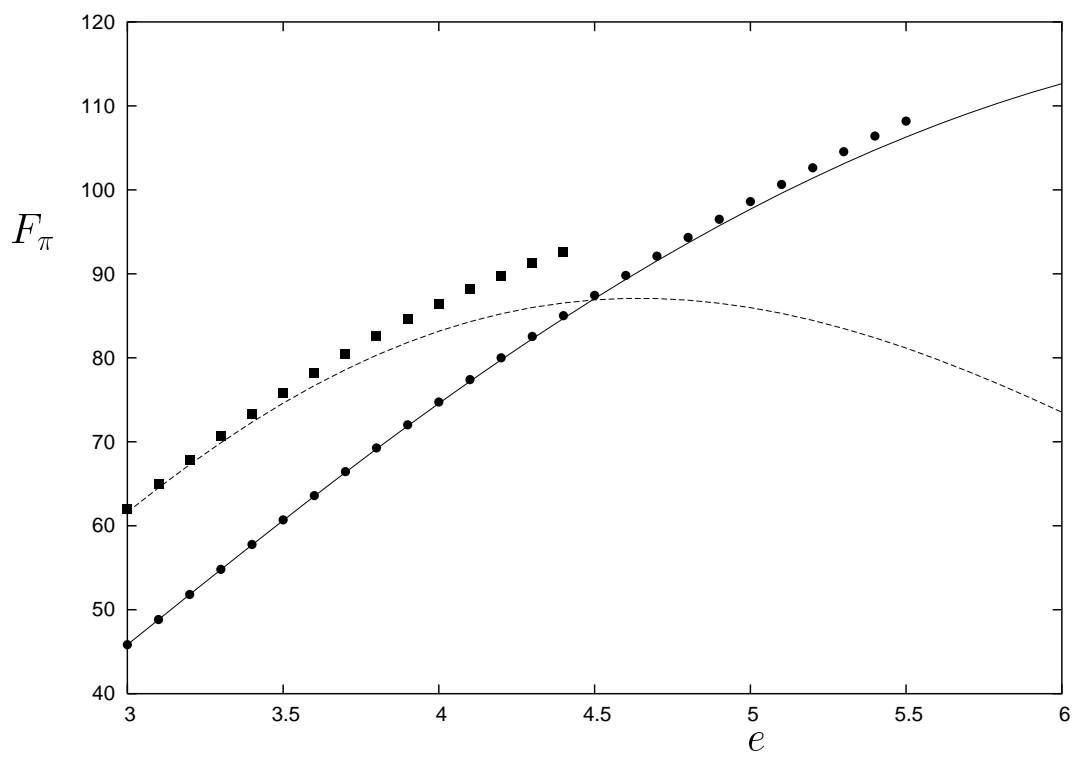

Figure 2: As Fig. 1 but with $m_{\pi}=276 \mathrm{MeV}$. Note that the data for the axial deformation of the nucleon and delta still do not cross.

In Fig. 2 we display (using the same notation as in Fig. 1) the results of both the rigid body and axial calculations with the pion mass set at twice the experimental value $m_{\pi}=276 \mathrm{MeV}$. Note that now the axial computations show some differences from the rigid body approximation when the delta spins close to its allowed limit, indicating an increased deformation from the static Skyrmion. Although the nucleon and delta data are now closer to an intersection, they still do not cross, so again there are no values of the Skyrme parameters to fit both masses.

An examination of the spinning Skyrmion configuration reveals that most of the deformation can be captured within a hedgehog ansatz, so the leading correction is associated with a deformation of the size of the Skyrmion. However, if the deformed hedgehog approximation of Refs. $[12,7]$ is implemented the nucleon and delta curves actually cross for this value of the pion mass, as the termination points are not correctly predicted.

Increasing the pion mass a little more, to two and a half times the experimental value $m_{\pi}=345 \mathrm{MeV}$, produces the data shown in Fig. 3. Now the axial delta data is substantially different from the rigid body approximation, and the nucleon and delta curves intersect. With this value of the pion mass the Skyrme parameters can be determined by fitting to the nucleon and delta masses and the parameters are found to be $e=4.90$ and $F_{\pi}=90.5$. This corresponds to a rescaled pion mass of $m=1.56$, which is remarkably close to the value required in the study of the Roper resonance [6].

It is interesting that the above values of $e$ and $F_{\pi}$ are similar to those of the standard parameters, but now require a pion mass which is more than twice the experimental value. 


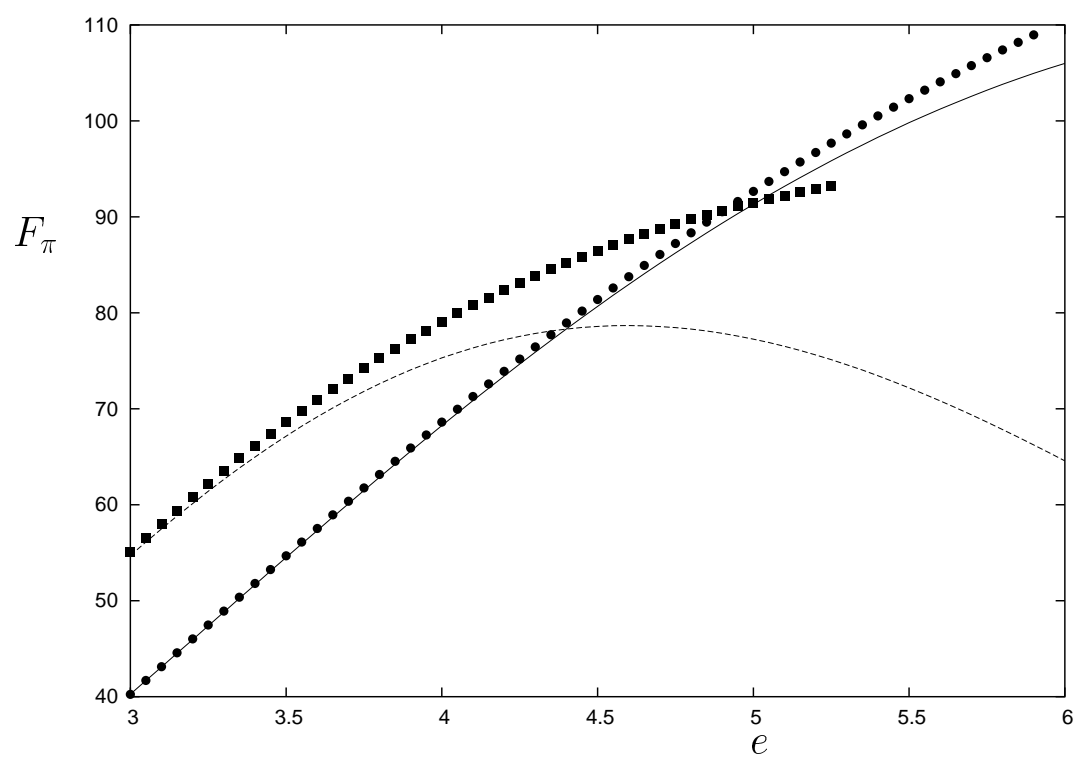

Figure 3: As Fig. 1 but with $m_{\pi}=345 \mathrm{MeV}$. Note that the data for the axial deformation of the nucleon and delta now cross.

There is clearly a critical pion mass in the range $276 \mathrm{MeV} \leq m_{\pi} \leq 345 \mathrm{MeV}$ above which a fit to the nucleon and delta masses is possible and below which it is not, but we have not computed this since it requires a substantial number of simulations and is probably not the best way to fit the Skyrme parameters. The values of $e$ and $F_{\pi}$ at the crossing point (when it exists) appear to be fairly stable to changes in the pion mass, so the above set of values seems a suitable set for further investigations.

In comparing our results with previous studies, we note that Skyrmions spin rather slowly in the sense that the deformation from the static Skyrmion is quite small even when the rotational frequency takes its maximal value. Only for a large pion mass (which sets the maximal rotation frequency) and then only for the delta, is a significant deformation found. This agrees with the findings of Ref.[14] in the sense that there it was also found that substantial axial deformations occur only for large values of the spin. However, we find that such solutions can not exist as finite energy spinning Skyrmions unless the Skyrme parameters are substantially different from the standard values.

Our results should be contrasted with similar studies on planar baby Skyrmions [10], where the important difference is that the moment of inertia of a baby Skyrmion is infinite as the maximal rotation frequency is reached, thus allowing any spin to be attained. In this case the deformation close to the allowed maximal spin is substantial, as one would expect from a diverging moment of inertia. 


\section{Conclusion}

In this paper we have used an axially symmetric numerical code to compute the energies of spinning Skyrmions and have determined the parameters of the Skyrme model to fit these energies to the masses of the nucleon and delta. We have described how the standard Skyrme parameters are obtained in a region which is not physical and are an artifact of the rigid body approximation. Moreover, we have shown that the nucleon and delta masses can be matched only if the pion mass is set at more than twice its experimental value. This result supports recent work [4] on multi-Skyrmions, which finds that setting the pion mass to be substantially larger than its experimental value yields multi-Skyrmions whose qualitative features are closer to those of real nuclei.

A valid criticism of fitting the Skyrme parameters to the nucleon and delta masses is that the delta is an unstable resonance, which nevertheless must be modeled as a stable spinning Skyrmion. Note that using the rigid body approximation, or the axial ansatz with a rotation frequency above the pion mass, does not model the delta as an unstable solution since there is simply no solution, rather than an unstable one. It would therefore seem that a better approach to fixing the Skyrme parameters is to use properties of multi-Skyrmions, and these appear to favour a larger pion mass [4]. The main result of the present paper is to conclude that a large pion mass is not incompatible with studies of the single Skyrmion, and indeed the nucleon and delta calculations support this view.

Finally, the parameter $m_{\pi}$ is interpreted as the pion mass but there are all kinds of quantization issues beyond the zero-mode quantization, such as the calculation of the Casimir energy [9], which are difficult to resolve due to the fact that the Skyrme model is a non-renormalizable field theory. As we have mentioned earlier, one optimistic approach is that these effects can be partially modeled by a renormalization of the Skyrme parameters, in which case $m_{\pi}$ could be interpreted as a renormalized pion mass, and therefore a larger value is not necessarily in conflict with the smaller experimental value.

\section{Acknowledgements}

Many thanks to Juan Ponciano, Bernd Schroers and Nick Manton for useful discussions. This work was supported by the PPARC special purpose grant "Classical Lattice Field Theory". SK acknowledges the EPSRC for a postdoctoral fellowship GR/S29478/01.

\section{References}

[1] G. S. Adkins and C. R. Nappi, The Skyrme model with pion masses, Nucl. Phys. B233, 109 (1984).

[2] G. S. Adkins, C. R. Nappi and E. Witten, Static properties of nucleons in the Skyrme model, Nucl. Phys. B228, 552 (1983). 
[3] C. Barnes, W. Baskerville and N. Turok, Normal modes of the $B=4$ Skyrme soliton, Phys. Rev. Lett. 79, 367 (1997); Normal mode spectrum of the deuteron in the Skyrme model, Phys. Lett. B411, 180 (1997).

[4] R. A. Battye and P. M. Sutcliffe, Skyrmions and the pion mass, Nucl. Phys. B705, $384(2005)$.

[5] E. Braaten and J. P. Ralston, Limitations of a semiclassical treatment of the Skyrme soliton, Phys. Rev. D31, 598 (1985).

[6] J. D. Breit and C. R. Nappi, Phase shifts of the Skyrmion breathing mode, Phys. Rev. Lett. 53, 889 (1984).

[7] N. Dorey, J. Hughes and M. P. Mattis, Skyrmion quantization and the decay of the delta, Phys. Rev. D50, 5816 (1994).

[8] S. Krusch and P. M. Sutcliffe, Sphalerons in the Skyrme model, J. Phys. A37, 9037 (2004).

[9] F. Meier and H. Walliser, Quantum corrections to baryon properties in chiral soliton models, Phys. Reports 289, 383 (1997).

[10] B. M. A. G. Piette, B. J. Schroers and W. J. Zakrzewski, Dynamics of baby Skyrmions, Nucl. Phys. B439, 205 (1995).

[11] R. Rajaraman, H. M. Sommermann, J. Wambach and H. W. Wyld, Stability of the rotating Skyrmion, Phys. Rev. D33, 287 (1986).

[12] B. J. Schroers, Dynamics of moving and spinning Skyrmions, Z. Phys. C61, 479 (1994).

[13] T. H. R. Skyrme, A nonlinear field theory, Proc. R. Soc. Lond. A260, 127 (1961).

[14] J. Wambach, H. W. Wyld and H. M. Sommermann, Axially deformed Skyrmions in a constrained variational approach, Phys. Lett. B186, 272 (1987).

[15] E. Witten, Global aspects of current algebra, Nucl. Phys. B223, 422 (1983); Current algebra, baryons, and quark confinement, ibid B223, 433 (1983). 